\title{
A sufficient condition for the continuity of solutions to a logarithmic diffusion equation
}

\author{
Naian Liao*
}

\begin{abstract}
This note gives a first sufficient condition that insures a non-negative, locally bounded, local solution to a logarithmically singular parabolic equation is continuous at a vanishing point and an estimate of the modulus of continuity is given. Moreover, an estimate of the Hausdorff measure of the set of discontinuity is established.
\end{abstract}

AMS Subject Classification (2010): Primary 35K67, 35B65; Secondary 35B45

Key Words: logarithmic diffusion, singular parabolic equations, continuity, Hausdorff measure

\section{Introduction and Main Results}

Let $E$ be an open set in $\mathbb{R}^{N}$. For $T>0$, let $E_{T}$ denote the cylindrical domain $E \times(0, T]$. Consider the quasi-linear, parabolic differential equation

$$
u_{t}-\Delta \ln u=0 \quad \text { weakly in } E_{T} .
$$

This equation is singular since its modulus of ellipticity $u^{-1} \rightarrow \infty$ as $u \rightarrow 0$. A non-negative function $u$ satisfying

$$
u \in C_{l o c}\left(0, T ; L_{l o c}^{2}(E)\right), \quad \ln u \in L_{l o c}^{2}\left(0, T ; W_{l o c}^{1,2}(E)\right)
$$

is called a local, weak sub(super)-solution to (1.1) if for every compact set $K \subset E$ and every sub-interval $\left[t_{1}, t_{2}\right] \subset(0, T]$

$$
\left.\int_{K} u \varphi d x\right|_{t_{1}} ^{t_{2}}+\int_{t_{1}}^{t_{2}} \int_{K}\left(-u \varphi_{t}+\frac{D u}{u} D \varphi\right) d x d t \leq(\geq) 0
$$

for all non-negative testing functions

$$
\varphi \in W_{l o c}^{1,2}\left(0, T ; L^{2}(K)\right) \cap L_{l o c}^{2}\left(0, T ; W_{o}^{1,2}(K)\right) .
$$

*Supported by Chongqing University Grant No. 106112015CDJXY100006 
A function $u$ that is both a local, weak sub-solution and a local, weak supersolution is a local, weak solution.

For $\rho>0$ we denote by $K_{\rho}(y)$ the cube centered at $y$ with side length $\rho$. If $y=0$ we use $K_{\rho}$. For $\theta>0$ introduce the cylinder with "vertex" at $(0,0)$

$$
Q_{\rho}(\theta)=K_{\rho} \times\left(-\theta \rho^{2}, 0\right] .
$$

If $\theta=1$ we use $Q_{\rho}$. Also a cylinder with "vertex" at $(y, s)$ is

$$
(y, s)+Q_{\rho}(\theta)=K_{\rho}(y) \times\left(s-\theta \rho^{2}, s\right] .
$$

Assume $u$ is a locally bounded, local solution. Let us suppose $\rho>0$ is so small that the cylinder $(y, s)+Q_{\rho} \subset E_{T}$. Up to a translation we may assume $(y, s)=(0,0)$ and let

$$
\omega=\underset{Q_{\rho}}{\operatorname{ess} \operatorname{osc} u} \text {. }
$$

Without loss of generality we assume $\omega \leq 1$ such that

$$
Q_{\rho}(\omega) \subset Q_{\rho} \quad \text { and } \quad \underset{Q_{\rho}(\omega)}{\operatorname{essosc}} u \leq \omega .
$$

Suppose in addition to the notion of solution that

$$
D \ln u \in L_{l o c}^{p}\left(E_{T}\right) \quad \text { for some } p>\frac{N+2}{2} .
$$

Note that when $N=1$ the integrability condition (1.2) is inherent in the notion of solution while in other cases it has to be imposed. Accordingly we define the quantity

$$
I_{p, \rho}(y, s)=\rho\left(\int_{(y, s)+Q_{\rho}}|D \ln u|^{p} d x d t\right)^{\frac{1}{p}}
$$

and $I_{p, \rho}=I_{p, \rho}(0,0)$. Then we have the following main theorem.

Theorem 1.1 Let $u$ be a non-negative, locally bounded, local solution to (1.1) and assume (1.2) is satisfied. Then there exist constants $\bar{C}>1$ and $\alpha \in(0,1)$ depending only on $N$, such that for any $\mu \in(0,1)$ and $0<r<\rho \leq R_{o}$ we have

$$
\underset{Q_{r}(\omega)}{\operatorname{ess} \operatorname{osc}} u \leq \bar{C}\left[\omega\left(\frac{r}{R_{o}}\right)^{(1-\mu) \alpha}+I_{p, R_{o}^{1-\mu} r^{\mu}}\right]
$$

In particular, the solution $u$ is continuous at the origin provided

$$
\limsup _{r \rightarrow 0} I_{p, r}=0 .
$$

Remark 1.1 Strictly speaking, we need the convention that the function $\rho \rightarrow$ $I_{p, \rho}$ is non-decreasing. In order to validate that, we need only to take

$$
\tilde{I}_{p, \rho}=\sup _{0<\tau<\rho} I_{p, \tau}
$$

in Theorem 1.1 
Remark 1.2 For $\lambda \geq 0, T>0$ and $N \geq 3$ the explicit solution

$$
u(x, t)=\frac{2(N-2)(T-t)^{\frac{N}{N-2}}}{\lambda+(T-t)^{\frac{2}{N-2}}|x|^{2}}
$$

is continuous up to its extinction time $T$. One verifies that when $\lambda>0$ and for any fixed $x_{o}$, there is a positive constant $C\left(x_{o}, \lambda, N, p\right)$ such that

$$
I_{p, r}\left(x_{o}, T\right) \leq C r^{\frac{4}{(N-2) p}+2} \rightarrow 0 \quad \text { as } r \rightarrow 0 .
$$

When $\lambda=0$, it gives an unbounded solution which, in particular, is discontinuous at $x=0$. Condition (1.3) is verified everywhere except for $x=0$. A direct calculation shows that

$$
D \ln u \in L_{l o c}^{p}\left(\mathbb{R}^{N} \times \mathbb{R}_{+}\right) \quad \text { for any } \frac{N+2}{2}<p<N .
$$

Furthermore, there exists some positive constant $C(N, p)$ such that for every $t<T$

$$
I_{p, r}(t, 0)=\left\{\begin{array}{l}
C(N, p), \quad \frac{N+2}{2}<p<N \\
\infty, \quad p \geq N .
\end{array}\right.
$$

Hence the condition (1.2) alone is not sufficient to ensure continuity.

Now define the set $S \subset E_{T}$ to consist of all discontinuous points of a local solution $u$ and

$$
S_{o}=\left\{(y, s) \in E_{T}: \limsup _{\rho \rightarrow 0} \frac{1}{\rho^{N+2-p}} \iint_{(y, s)+Q_{\rho}}|D \ln u|^{p} d x d t>0\right\} .
$$

As a direct consequence of Theorem 1.1 it is straightforward to see that $S \subset S_{o}$. Moreover we are going to obtain an estimate of the Hausdorff measure of the set $S_{o}$.

The parabolic Hausdorff measure $P_{k}$ is defined in a way similar to the usual Hausdorff measure $H_{k}$ but using the parabolic metric on $\mathbb{R}^{N} \times \mathbb{R}$. For any set $U \subset \mathbb{R}^{N} \times \mathbb{R}$ and $k \geq 0$ we define

$$
P_{k}(U)=\lim _{\delta \rightarrow 0} P_{k}^{\delta}(U),
$$

where

$$
P_{k}^{\delta}(U)=\inf \left\{\sum_{i=1}^{\infty} r_{i}^{k}: U \subset \bigcup_{i}\left[\left(y_{i}, t_{i}\right)+Q_{r_{i}}\right], r_{i}<\delta\right\} .
$$

$P_{k}$ so defined is an outer measure whose $\sigma$-algebra contains all Borel sets of $\mathbb{R}^{N} \times \mathbb{R}$ (Chapter 2, [10]). It should be pointed out that the parabolic Hausdorff measure dominates the usual Hausdorff measure in the sense that there is some constant $C(N, k)$ such that for any subset $U$ of $\mathbb{R}^{N} \times \mathbb{R}$ one has

$$
H_{k}(U) \leq C P_{k}(U) .
$$

Regarding the Hausdorff measure of the discontinuity set we have the following consequence of Theorem 1.1 . 
Theorem 1.2 Let $u$ be a non-negative, locally bounded, local solution to (1.1) and assume (1.2) is satisfied. Then we have

$$
\begin{aligned}
& P_{N+2-p}\left(S_{o}\right)=0, \quad N>1 \quad \text { and } \\
& P_{1}\left(S_{o}\right)=0, \quad N=1 .
\end{aligned}
$$

Remark 1.3 When $N=1$ the possible discontinuous points of a non-negative, locally bounded, local solution to (1.1) cannot occupy a line in $\mathbb{R}^{2}$. Generally one gets less discontinuity as the $L^{p}$ integrability of $D \ln u$ increases and eventually, the solution is continuous at every point if one has $p \geq N+2$.

\subsection{Novelty and Significance}

Equation (1.1) describes the evolution of the Ricci flow for complete $\mathbb{R}^{2}$ ([18]). It also arises from modeling the thickness of a viscous liquid thin film that lies on a rigid plate under the influence of the van der Waals force ([17]).

Physical and geometric motivations of (1.1) make sense mainly for $N=$ 2 , but the problem is intriguing in the effort to shed light on the structural properties of singular diffusion equations.

Questions concerning both existence and non-existence of solutions to the Cauchy problem of (1.1) and its related elliptic equation are investigated in [1, 2, 4, 5, 12, 11, 16, (just mention few).

The study of local behavior of local solutions to (1.1) has been initiated in [6. 7. Equation (1.1) can be viewed as a formal limit of the porous medium equation

$$
u_{t}-\operatorname{div}\left(u^{m-1} D u\right)=0 \quad \text { as } m \rightarrow 0 .
$$

A proof of Hölder continuity for non-negative, locally bounded, local solutions to the porous medium equation can be found in Appendix B of 9 . However, the local behavior of local solutions to (1.1) presents many striking differences from that of local solutions to the porous medium equation. See [14 for more detailed discussion.

It was shown in [6] that if one assumes that

$$
u \in L_{l o c}^{r}\left(E_{T}\right) \quad \text { for some } \quad r>\max \left\{1, \frac{N}{2}\right\},
$$

then $u$ is locally bounded. If in addition one assumes that

$$
\ln u \in L_{l o c}^{\infty}\left(0, T ; L_{l o c}^{p}(E)\right) \text { for some } \quad p>N+2,
$$

then a Harnack-type inequality is established and thus, if the solution does not vanish identically on a hyperplane normal to the time axis, then the equation (1.1) is neither degenerate nor singular in a backward cylinder with its vertex on the hyperplane. As a result $u$ is a classical solution in such a cylinder. In fact, it is shown in [8] that under such circumstances the solution is analytic in space variables while infinitely differentiable in time.

Nevertheless, these results do not explain why some explicit solutions, (1.4) for example, could be continuous up to their extinction time. Theorem 1.1 
gives a first sufficient condition that insures continuity at a vanishing point of $u$, and an explicit estimate of the modulus of continuity is given. Moreover, we establish in Theorem 1.2 an estimate on the Hausdorff measure of the set of discontinuity of $u$.

Those effort being made, it is interesting to ask whether the higher integrability conditon (1.2) of $D \ln u$ for $N>1$ can be obtained from the notion of solution and whether the condition (1.3) is necessary for a point to be a continuity point of $u$. Last but not least, can we construct an explicit bounded solution with discontinuity? In [15] when $N=1$, a solution discontinuous on a line segment was constructed. However, the notion of solution used seems different from this note, since our results indicate such a phenomenon is not allowed for our solutions.

Acknowledgement. This paper was finalized during my visit to Vanderbilt University in November 2016. I am grateful for many helpful discussions with Professor Emmanuele DiBenedetto and Professor Ugo Gianazza. Professor Gianazza also read carefully the early version of this paper and came up with a lot of valuable comments. I am really indebted to both of them.

\section{Proof of Theorem 1.2 Assuming Theorem 1.1}

The proof of Theorem 1.2 is based on the following

Proposition 2.1 Let $f \in L_{l o c}^{1}\left(\mathbb{R}^{N+1}\right)$, suppose $0 \leq s<N+2$ and define

$$
\Lambda_{s}=\left\{(y, s) \in \mathbb{R}^{N+1}: \limsup _{\rho \rightarrow 0} \frac{1}{\rho^{s}} \iint_{(y, s)+Q_{\rho}}|f| d x d t>0\right\} .
$$

Then

$$
P_{s}\left(\Lambda_{s}\right)=0
$$

This is a parabolic counterpart of a similar result shown in [10] (Theorem 3, p.77). Now we are ready to present

Proof of Theorem 1.2, When $N>1$, since we assume

$$
D \ln u \in L_{l o c}^{p}\left(E_{T}\right) \text { for some } p>\frac{N+2}{2},
$$

a straightforward application of Proposition 2.1 yields the desired conclusion.

When $N=1$, the notion of solution gives

$$
D \ln u \in L_{l o c}^{2}\left(E_{T}\right)
$$

and by the Hölder inequality with $p<2$

$$
\left[\frac{1}{\rho^{3-p}} \iint_{Q_{\rho}}|D \ln u|^{p} d x d t\right]^{\frac{1}{p}} \leq\left[\frac{1}{\rho} \iint_{Q_{\rho}}|D \ln u|^{2} d x d t\right]^{\frac{1}{2}} .
$$


Thus

$$
S_{o} \subset\left\{(y, s) \in E_{T}: \limsup _{\rho \rightarrow 0} \frac{1}{\rho} \iint_{(y, s)+Q_{\rho}}|D \ln u|^{2} d x d t>0\right\}
$$

and again by Proposition 2.1 we obtain

$$
P_{1}\left(S_{o}\right)=0 .
$$

This finishes the proof.

The rest of the note is devoted to proving Theorem 1.1 .

\section{Some Preliminary Estimates}

\subsection{Energy Estimates}

Proposition 3.1 Let $u$ be a local, weak super-solution to (1.1). Then there is a positive constant $\gamma$ depending only on $N$ such that for every cylinder $(y, s)+$ $Q_{\rho}(\theta) \subset E_{T}$, every $k \in \mathbb{R}_{+}$, and every non-negative, piecewise smooth cutoff function $\zeta$ vanishing on $\partial K_{\rho}(y)$,

$$
\begin{aligned}
\underset{s-\theta \rho^{2}<t<s}{\operatorname{ess} \sup ^{2}} & \frac{1}{2} \int_{K_{\rho}(y)}(u-k)_{-}^{2} \zeta^{2} d x+k^{-1} \iint_{(y, s)+Q_{\rho}(\theta)}\left|D\left[(u-k)_{-} \zeta\right]\right|^{2} d x d t \\
& \leq \frac{1}{2} \int_{K_{\rho}(y)}(u-k)_{-}^{2} \zeta^{2}\left(x, s-\theta \rho^{2}\right) d x \\
& +\iint_{(y, s)+Q_{\rho}(\theta)}(u-k)_{-}^{2} \zeta\left|\zeta_{t}\right| d x d t \\
& +k^{-1} \iint_{(y, s)+Q_{\rho}(\theta)}(u-k)_{-}^{2}|D \zeta|^{2} d x d t \\
& +2 \iint_{(y, s)+Q_{\rho}(\theta)}|D \ln u|(u-k)_{-}|D \zeta| \zeta d x d t .
\end{aligned}
$$

Proof. We may assume $(y, s)=(0,0)$. In the weak formulation for supersolutions to (1.1), we take the test function

$$
\varphi=-(u-k)_{-} \zeta^{2}
$$

over the cylinder

$$
Q_{t}=K_{\rho} \times\left(-\theta \rho^{2}, t\right] \quad \text { for } \quad t \in\left(-\theta \rho^{2}, 0\right],
$$

modulo a standard Steklov averaging process. This gives

$$
-\iint_{Q_{t}} u_{\tau}(u-k)_{-} \zeta^{2} d x d \tau-\iint_{Q_{t}} \zeta^{2} D \ln u D(u-k)_{-} d x d \tau
$$




$$
=\iint_{Q_{t}} 2 \zeta(u-k)_{-} D \ln u D \zeta d x d \tau .
$$

The first term on the left-hand side is estimated by

$$
\begin{aligned}
& -\iint_{Q_{t}} u_{\tau}(u-k)_{-} \zeta^{2} d x d \tau \\
& \geq \frac{1}{2} \int_{K_{\rho}}(u-k)_{-}^{2} \zeta^{2}(x, t) d x-\frac{1}{2} \int_{K_{\rho}}(u-k)_{-}^{2} \zeta^{2}\left(x,-\theta \rho^{2}\right) d x \\
& \quad-\iint_{Q_{\rho}(\theta)}(u-k)_{-}^{2} \zeta\left|\zeta_{\tau}\right| d x d \tau
\end{aligned}
$$

while the second term is estimated by

$$
-\iint_{Q_{t}} \zeta^{2} D \ln u D(u-k)_{-} d x d \tau \geq k^{-1} \iint_{Q_{\rho}(\theta)}\left|D(u-k)_{-}\right|^{2} \zeta^{2} d x d \tau .
$$

Next the term on the right side is

$$
\iint_{Q_{t}} 2 \zeta(u-k)_{-} D \ln u D \zeta d x d \tau \leq 2 \iint_{Q_{\rho}(\theta)}|D \ln u|(u-k)_{-}|D \zeta| \zeta d x d \tau
$$

Combining all these estimates yields the conclusion.

Proposition 3.2 Let $u$ be a local, weak sub-solution to (1.1) in $E_{T}$. There exists a positive constant $\gamma=\gamma(N)$, such that for every cylinder $(y, s)+Q_{\rho}(\theta) \subset$ $E_{T}$, every $k \in \mathbb{R}_{+}$, and every non-negative, piecewise smooth cutoff function $\zeta$ vanishing on $\partial K_{\rho}(y)$,

$$
\begin{aligned}
& \underset{s-\theta \rho^{2}<t \leq s}{\operatorname{ess} \sup _{s}} \int_{K_{\rho}(y)}(u-k)_{+}^{2} \zeta^{2}(x, t) d x \\
& \quad-\int_{K_{\rho}(y)}(u-k)_{+}^{2} \zeta^{2}\left(x, s-\theta \rho^{2}\right) d x \\
& \quad+\iint_{(y, s)+Q_{\rho}(\theta)} \frac{\left|D\left[(u-k)_{+} \zeta\right]\right|^{2}}{u} d x d t \\
& \leq \gamma \iint_{(y, s)+Q_{\rho}(\theta)}(u-k)_{+}^{2} \zeta\left|\zeta_{t}\right| d x d t \\
&+\gamma \iint_{(y, s)+Q_{\rho}(\theta)} \frac{(u-k)_{+}^{2}}{u}|D \zeta|^{2} d x d t .
\end{aligned}
$$

Proof. After a translation may assume $(y, s)=(0,0)$. Take the test function $\varphi=(u-k)_{+} \zeta^{2}$ over $Q_{t}$ modulo a standard Steklov averaging process, and perform standard calculations. The various integrals are extended over the set $[u>k]$ and since $k>0$, they are all well defined. 


\subsection{A Logarithmic Estimate for Sub-Solutions}

Introduce the logarithmic function

$$
\psi(u)=\ln ^{+}\left[\frac{H}{H-(u-k)_{+}+c}\right]
$$

where

$$
H=\operatorname{essip}_{(y, s)+Q_{\rho}(\theta)}(u-k)_{+}, \quad 0<c<\min \{1 ; H\}
$$

and for $s>0$

$$
\ln ^{+} s=\max \{\ln s ; 0\} .
$$

In the cylinder $(y, s)+Q_{\rho}(\theta)$ take a non-negative, piecewise smooth cutoff function $\zeta$ independent of $t$.

Proposition 3.3 Let $u$ be a non-negative, locally bounded, local, weak subsolution to equation (1.1) in $E_{T}$. There exists a constant $\gamma$, depending only on the $N$, such that for every cylinder

$$
(y, s)+Q_{\rho}(\theta) \subset E_{T}
$$

and for every level $k \geq 0$ we have

$$
\begin{aligned}
& \sup _{s-\theta \rho^{2}<t<s} \int_{K_{\rho}(y)} \psi^{2}(u)(x, t) \zeta^{2}(x) d x \\
& \leq \int_{K_{\rho}(y)} \psi^{2}(u)\left(x, s-\theta \rho^{2}\right) \zeta^{2}(x) d x+\gamma \iint_{(y, s)+Q_{\rho}(\theta)} \frac{\psi(u)}{u}|D \zeta|^{2} d x d t .
\end{aligned}
$$

Proof. Take $(y, s)=(0,0)$ and work within the cylinder $Q_{t}$ introduced before in the energy estimates. In the weak formulation of (1.1) take the testing function

$$
\varphi=\frac{\partial}{\partial u}\left[\psi^{2}(u)\right] \zeta^{2}=2 \psi \psi^{\prime} \zeta^{2}
$$

By direct calculation

$$
\left[\psi^{2}(u)\right]^{\prime \prime}=2(1+\psi) \psi^{\prime 2} \in L_{l o c}^{\infty}\left(E_{T}\right)
$$

which implies that such a $\varphi$ is an admissible testing function, modulo a Steklov averaging process. Since $\psi(u)$ vanishes on the set where $(u-k)_{+}=0$

$$
\iint_{Q_{t}} u_{\tau}\left[\psi^{2}\right]^{\prime} \zeta^{2} d x d t=\int_{K_{\rho}} \psi^{2}(x, t) \zeta^{2} d x-\int_{K_{\rho}} \psi^{2}\left(x,-\theta \rho^{2}\right) \zeta^{2} d x .
$$

As for the remaining term

$$
\begin{aligned}
& \iint_{Q_{t}} \frac{D u}{u} \cdot D \varphi d x d \tau \\
& \geq 2 \iint_{Q_{t}}(1+\psi) \psi^{\prime 2} \frac{|D u|^{2}}{u} \zeta^{2} d x d \tau-4 \iint_{Q_{t}} \frac{|D u|}{u} \psi \psi^{\prime} \zeta|D \zeta| d x d \tau \\
& \geq \iint_{Q_{t}}(1+\psi) \psi^{\prime 2} \frac{|D u|^{2}}{u} \zeta^{2} d x d \tau-\gamma \iint_{Q_{t}} \frac{\psi}{u}|D \zeta|^{2} d x d \tau
\end{aligned}
$$

Collecting these estimates establishes the proposition. 


\section{DeGiorgi-type Lemmas}

For a cylinder $(y, s)+Q_{2 \rho}(\theta) \subset E_{T}$ denote by $\mu_{ \pm}$and $\omega$, numbers satisfying

$$
\mu_{+} \geq \underset{\left[(y, s)+Q_{2 \rho}(\theta)\right]}{\operatorname{ess} \sup } u, \quad \mu_{-} \leq \underset{\left[(y, s)+Q_{2 \rho}(\theta)\right]}{\operatorname{essinf}} u, \quad \omega=\mu_{+}-\mu_{-} .
$$

Denote by $\xi$ and $a$ fixed numbers in $(0,1)$.

Lemma 4.1 Let u be a non-negative, locally bounded, local, weak super-solution to (1.1). Then there is a constant $\nu_{-}$depending on the data and $\theta, \xi, \omega$, a, such that if

$$
\left|\left[u \leq \mu^{-}+\xi \omega\right] \cap\left[(y, s)+Q_{2 \rho}(\theta)\right]\right| \leq \nu_{-}\left|Q_{2 \rho}(\theta)\right|,
$$

then either

$$
\xi \omega \leq I_{p, \rho}(y, s)
$$

or

$$
u \geq \mu^{-}+a \xi \omega \quad \text { a.e. in }(y, s)+Q_{\frac{\rho}{2}}(\theta) .
$$

Proof. We may take $(y, s)=(0,0)$. Set

$$
\rho_{n}=\rho+\frac{\rho}{2^{n}}, \quad K_{n}=K_{\rho_{n}}, \quad Q_{n}=K_{n} \times\left(-\theta \rho_{n}^{2}, 0\right]
$$

Consider a non-negative, piecewise smooth cutoff function on $Q_{n}$ of the form $\zeta(x, t)=\zeta_{1}(x) \zeta_{2}(t)$, where

$$
\begin{aligned}
& \zeta_{1}=\left\{\begin{array}{lll}
1 & \text { in } K_{n+1} & \left|D \zeta_{1}\right| \leq \frac{1}{\rho_{n}-\rho_{n+1}}=\frac{2^{n+1}}{\rho} \\
0 & \text { in } \mathbb{R}^{N}-K_{n} & 0 \leq \zeta_{2, t} \leq \frac{1}{\theta\left(\rho_{n}^{2}-\rho_{n+1}^{2}\right)} \leq \frac{2^{2(n+1)}}{\theta \rho^{2}}
\end{array}\right. \\
& \zeta_{2}=\left\{\begin{array}{lll}
0 & \text { for } t<-\theta \rho_{n}^{2} & \text { for } t \geq-\theta \rho_{n+1}^{2}
\end{array}\right.
\end{aligned}
$$

Now apply the energy estimate to $\left(u-k_{n}\right)_{-}$in the cylinder $Q_{n}$ with

$$
k_{n}=\mu^{-}+a \xi \omega+\frac{1-a}{2^{n}} \xi \omega
$$

to obtain

$$
\begin{aligned}
\underset{-\theta \rho^{2}<t<0}{\operatorname{ess} \sup _{K_{n}}} & \int_{K_{n}}\left(u-k_{n}\right)_{-}^{2} \zeta^{2}(x, t) d x+\frac{1}{\mu^{-}+\xi \omega} \iint_{Q_{n}}\left|D\left[\left(u-k_{n}\right)_{-} \zeta\right]\right|^{2} d x d t \\
& \leq \frac{4^{n}}{\theta \rho^{2}} \iint_{Q_{n}}\left(u-k_{n}\right)_{-}^{2} d x d t+\frac{4^{n}}{\left(\mu^{-}+a \xi \omega\right) \rho^{2}} \iint_{Q_{n}}\left(u-k_{n}\right)_{-}^{2} d x d t \\
& +4^{n} \frac{M \xi \omega}{\rho}\left|\left[u<k_{n}\right] \cap Q_{n}\right|^{1-\frac{1}{p}}
\end{aligned}
$$


where

$$
M=\left(\iint_{Q_{\rho}}|D \ln u|^{p} d x d t\right)^{\frac{1}{p}} .
$$

Let $A_{n}=\left[u<k_{n}\right] \cap Q_{n}$. By the standard parabolic embedding theorem (Proposition 3.1, Chapter 1 of [3]), we obtain

$$
\begin{aligned}
& \left(\frac{1-a}{2^{n+1}} \xi \omega\right)^{2}\left|A_{n+1}\right| \\
& \leq \iint_{Q_{n}}\left(u-k_{n}\right)_{-}^{2} \zeta^{2} d x d t \\
& \leq\left(\iint_{Q_{n}}\left[\left(u-k_{n}\right)-\zeta\right]^{2 \frac{N+2}{N}} d x d t\right)^{\frac{N}{N+2}}\left|A_{n}\right|^{\frac{2}{N+2}} \\
& \leq\left(\iint_{Q_{n}} \mid D\left[\left.\left(u-k_{n}\right)_{-} \zeta\right|^{2} d x d t\right)^{\frac{N}{N+2}}\right. \\
& \times\left(\operatorname{ess~sup}_{-\theta \rho^{2}<t<0} \int_{K_{n}}\left(u-k_{n}\right)_{-}^{2} \zeta^{2}(x, t) d x\right)^{\frac{2}{N+2}}\left|A_{n}\right|^{\frac{2}{N+2}} \\
& \leq 4^{n}\left|A_{n}\right|^{\frac{2}{N+2}}\left(\mu^{-}+\xi \omega\right)^{\frac{N}{N+2}} \\
& \times\left[\left(\frac{1}{\theta}+\frac{1}{\mu^{-}+a \xi \omega}\right) \frac{(\xi \omega)^{2}}{\rho^{2}}\left|A_{n}\right|+\frac{M \xi \omega}{\rho}\left|A_{n}\right|^{1-\frac{1}{p}}\right] .
\end{aligned}
$$

Setting

$$
Y_{n}=\frac{\left|A_{n}\right|}{\left|Q_{n}\right|},
$$

we have

$$
\begin{aligned}
Y_{n+1} & \leq \frac{\gamma 4^{2 n}}{(1-a)^{2}}\left[\left(\left(\frac{\mu^{-}+\xi \omega}{\theta}\right)^{\frac{N}{N+2}}+\gamma_{o}\left(\frac{\theta}{\mu^{-}+a \xi \omega}\right)^{\frac{2}{N+2}}\right) Y_{n}^{1+\frac{2}{N+2}}\right. \\
& \left.+\frac{I_{p, \rho}}{\xi \omega}\left(\frac{\mu^{-}+\xi \omega}{\theta}\right)^{\frac{N}{N+2}} Y_{n}^{1-\frac{1}{p}+\frac{2}{N+2}}\right]
\end{aligned}
$$

where

$$
\gamma_{o}=\left(\frac{\mu^{-}+\xi \omega}{\mu^{-}+a \xi \omega}\right)^{\frac{N}{N+2}}
$$

Suppose $I_{p, \rho} \leq \xi \omega$; we have

$$
Y_{n+1} \leq \frac{\gamma 4^{2 n}}{(1-a)^{2}}\left[\left(\frac{\mu^{-}+\xi \omega}{\theta}\right)^{\frac{N}{N+2}}+\gamma_{o}\left(\frac{\theta}{\mu^{-}+a \xi \omega}\right)^{\frac{2}{N+2}}\right] Y_{n}^{1+\beta}
$$

where

$$
\beta=\frac{2}{N+2}-\frac{1}{p}>0 .
$$


It follows from Lemma 4.1 of Chapter 1 of [3] that $Y_{n}$ tend to 0 provided

$$
Y_{o} \leq \nu_{-} \stackrel{\text { def }}{=} A^{-\frac{1}{\beta}} 16^{-\frac{1}{\beta^{2}}}
$$

where

$$
A=\frac{\gamma}{(1-a)^{2}}\left[\left(\frac{\mu^{-}+\xi \omega}{\theta}\right)^{\frac{N}{N+2}}+\gamma_{o}\left(\frac{\theta}{\mu^{-}+a \xi \omega}\right)^{\frac{2}{N+2}}\right] .
$$

This finishes the proof.

Some remarks are in order.

Remark 4.1 Without loss of generality, we may assume that $\mu^{-}<\frac{1}{2} \xi \omega$. In such a case the quantity $A$ above reduces to

$$
A=\frac{\gamma}{(1-a)^{2}}\left[\left(\frac{\xi \omega}{\theta}\right)^{\frac{N}{N+2}}+\left(\frac{\theta}{a \xi \omega}\right)^{\frac{2}{N+2}}\right]
$$

Remark 4.2 The either-or conclusion is necessary. Without $\xi \omega>I_{p, \rho}(y, s)$, in general one cannot obtain

$$
u \geq \mu^{-}+a \xi \omega \quad \text { a.e. in }(y, s)+Q_{\frac{\rho}{2}}(\theta) .
$$

See Remark C.1 in Appendix $C$ of [14].

Lemma 4.2 Let u be a non-negative, locally bounded, local, weak sub-solution to equation (1.1), in $E_{T}$. Assume that

$$
\omega \geq \frac{1}{b+1} \mu_{+},
$$

for some positive parameter $b$ to be chosen later. There exists a positive number $\nu_{+}$, depending upon $\omega, \theta, \xi, a$ and $N$, such that if

$$
\left|\left[u \geq \mu_{+}-\xi \omega\right] \cap\left[(y, s)+Q_{2 \rho}(\theta)\right]\right| \leq \nu_{+}\left|Q_{2 \rho}(\theta)\right|
$$

then

$$
u \leq \mu_{+}-a \xi \omega \quad \text { a.e. in }(y, s)+Q_{\rho}(\theta) .
$$

Proof. Assume $(y, s)=(0,0)$ and for $n=0,1, \ldots$ set

$$
\rho_{n}=\rho+\frac{\rho}{2^{n}}, \quad K_{n}=K_{\rho_{n}}, \quad Q_{n}=K_{n} \times\left(-\theta \rho_{n}^{2}\right] .
$$

Let $\zeta$ be a non-negative, piecewise smooth cutoff function on $Q_{n}$ defined as in the previous lemma. Introduce the sequence of truncating levels

$$
k_{n}=\mu_{+}-\xi_{n} \omega \quad \text { with } \quad \xi_{n}=a \xi+\frac{1-a}{2^{n}} \xi,
$$


and write down the energy estimates (3.1) over the cylinder $Q_{n}$, for the truncated function $\left(u-k_{n}\right)_{+}$. Taking also into account (4.2), this gives

$$
\begin{aligned}
\underset{-\theta \rho_{n}^{2}<t \leq 0}{\operatorname{ess} \sup } \int_{K_{n}} & \left(u-k_{n}\right)_{+}^{2} \zeta^{2}(x, t) d x \\
& +\iint_{Q_{n}} \frac{\left|D\left[\left(u-k_{n}\right)_{+} \zeta\right]\right|^{2}}{u} d x d \tau \\
\leq & \gamma \frac{2^{2 n}}{\rho^{2}}(\xi \omega)^{2} \iint_{Q_{n}}\left(\frac{1}{(1-\xi) \omega}+\frac{1}{\theta}\right) \chi_{\left[u>k_{n}\right]} d x d \tau \\
\leq & \gamma \frac{2^{2 n}}{\rho^{2}}(\xi \omega)^{2} \frac{1}{(1-\xi) \omega}\left(1+\frac{\omega}{\theta}\right)\left|\left[u>k_{n}\right] \cap Q_{n}\right| .
\end{aligned}
$$

To estimate below the second integral on the left-hand side, take into account that $u \leq \mu_{+}$and (4.2). This gives

$$
\iint_{Q_{n}} \frac{\left|D\left[\left(u-k_{n}\right)_{+} \zeta\right]\right|^{2}}{u} d x d \tau \geq \frac{1}{(b+1) \omega} \iint_{Q_{n}}\left|D\left[\left(u-k_{n}\right)_{+} \zeta\right]\right|^{2} d x d \tau .
$$

Setting

$$
A_{n}=\left[u>k_{n}\right] \cap Q_{n} \quad \text { and } \quad Y_{n}=\frac{\left|A_{n}\right|}{\left|Q_{n}\right|},
$$

and combining these estimates gives

$$
\begin{aligned}
\operatorname{ess~sup}_{-\theta \rho_{n}^{2}<t \leq 0} \int_{K_{n}}(u & \left.-k_{n}\right)_{+}^{2} \zeta^{2}(x, t) d x \\
& +\frac{1}{(b+1) \omega} \iint_{Q_{n}}\left|D\left[\left(u-k_{n}\right)_{+} \zeta\right]\right|^{2} d x d \tau \\
\leq & \gamma \frac{2^{2 n}}{\rho^{2}} \frac{(\xi \omega)^{2}}{(1-\xi) \omega}\left(1+\frac{\omega}{\theta}\right)\left|A_{n}\right| .
\end{aligned}
$$

Apply Hölder's inequality and the embedding Proposition 3.1 of Chapter 1 of [3], and recall that $\zeta=1$ on $Q_{n+1}$, to get

$$
\begin{aligned}
&\left(\frac{1-a}{2^{n+1}}\right)^{2}(\xi \omega)^{2}\left|A_{n+1}\right| \leq \iint_{Q_{n+1}}\left(u-k_{n}\right)_{+}^{2} d x d \tau \\
& \leq\left(\iint_{Q_{n}}\left[\left(u-k_{n}\right)_{+} \zeta\right]^{\frac{N+2}{N}} d x d \tau\right)^{\frac{N}{N+2}}\left|A_{n}\right|^{\frac{2}{N+2}} \\
& \leq \gamma\left(\iint_{Q_{n}}\left|D\left[\left(u-k_{n}\right)_{+} \zeta\right]\right|^{2} d x d \tau\right)^{\frac{N}{N+2}} \\
& \times\left(\operatorname{esssup}_{-\theta \rho_{n}^{2}<t \leq 0} \int_{K_{n}}\left[\left(u-k_{n}\right)_{+} \zeta\right]^{2}(x, t) d x\right)^{\frac{2}{N+2}}\left|A_{n}\right|^{\frac{2}{N+2}}
\end{aligned}
$$

for a constant $\gamma$ depending only upon $N$. Combine this with (4.3) to get

$$
\left|A_{n+1}\right| \leq \frac{\gamma 2^{4 n}}{(1-a)^{2} \rho^{2}} \frac{(b+1)^{\frac{N}{N+2}}}{(1-\xi)} \frac{1}{\omega^{\frac{2}{N+2}}}\left(1+\frac{\omega}{\theta}\right)\left|A_{n}\right|^{1+\frac{2}{N+2}} .
$$


In terms of $Y_{n}=\frac{\left|A_{n}\right|}{\left|Q_{n}\right|}$ this can be rewritten as

$$
Y_{n+1} \leq \frac{\gamma 2^{4 n}(b+1)^{\frac{N}{N+2}}}{(1-a)^{2}(1-\xi)}\left(\frac{\theta}{\omega}\right)^{\frac{2}{N+2}}\left(1+\frac{\omega}{\theta}\right) Y_{n}^{1+\frac{2}{N+2}} .
$$

By Lemma 4.1 of Chapter 1 of [3], $\left\{Y_{n}\right\} \rightarrow 0$ as $n \rightarrow \infty$, provided

$$
Y_{o}=\frac{\left|A_{o}\right|}{\left|Q_{o}\right|} \leq\left[\frac{(1-a)^{2}(1-\xi)}{\gamma 4^{N+2}(b+1)^{\frac{N}{N+2}}}\right]^{\frac{N+2}{2}} \frac{\frac{\omega}{\theta}}{\left(1+\frac{\omega}{\theta}\right)^{\frac{N+2}{2}}} \stackrel{\text { def }}{=} \nu_{+} .
$$

This finishes the proof.

\section{Proof of Theorem 1.1}

Fix $\left(x_{o}, t_{o}\right) \in E_{T}$ and let $\rho>0$ be so small that $\left(x_{o}, t_{o}\right)+Q_{\rho} \subset E_{T}$; we may assume that $\left(x_{o}, t_{o}\right)$ coincides with the origin. Set

$$
\mu_{+}=\underset{Q_{\rho}}{\operatorname{ess} \sup } u, \quad \mu_{-}=\underset{Q_{\rho}}{\operatorname{essinf}} u, \quad \omega=\mu_{+}-\mu_{-} .
$$

Without loss of generality we may assume $\omega \leq 1$, such that

$$
Q_{\rho}(\omega) \subset Q_{\rho} \quad \text { and } \quad \underset{Q_{\rho}(\omega)}{\operatorname{ess} \operatorname{Osc}} u \leq \omega .
$$

The proof now unfolds along several cases.

\subsection{Case I}

First of all, let us suppose

$$
\mu_{-} \geq \frac{1}{8} \omega \quad \Leftrightarrow \quad \mu_{+} \leq 9 \mu_{-} .
$$

Without loss of generality we may assume $\mu_{+} \leq 1$ such that

$$
Q_{\rho}\left(\mu_{+}\right) \subset Q_{\rho} \quad \text { and } \quad \underset{Q_{\rho}\left(\mu_{+}\right)}{\operatorname{ess} \operatorname{osc}} u \leq \omega .
$$

Introduce the change of time variable and unknown function

$$
\tau=\mu_{+}^{-1} t \quad \text { and } \quad v(\cdot, \tau)=\frac{u(\cdot, t)}{\mu_{+}} .
$$

Then

$$
v_{\tau}-\operatorname{div} \frac{D v}{v}=0 \quad \text { weakly in } \quad Q_{\rho}
$$

with

$$
\frac{1}{9} \leq v \leq 1
$$


Thus, by the classical parabolic theory ([13]), there exists $\eta \in(0,1)$ depending only on $N$ such that

$$
\underset{Q_{\frac{\rho}{2}}}{\operatorname{ess} \operatorname{osc} v} \leq(1-\eta) \underset{Q_{\rho}}{\operatorname{ess} \operatorname{osc}} v .
$$

Returning to the original coordinates we conclude that

$$
\underset{Q_{\frac{\rho}{2}}}{\operatorname{ess} \operatorname{osc}} u \leq \underset{Q_{\frac{\rho}{2}}\left(\mu_{+}\right)}{\operatorname{ess} \operatorname{osc}} u \leq(1-\eta) \underset{Q_{\rho}\left(\mu_{+}\right)}{\operatorname{ess} \operatorname{osc}} u \leq(1-\eta) \omega .
$$

\subsection{Case II}

Now suppose

$$
\mu_{+}>9 \mu_{-}
$$

which is equivalent to

$$
\omega>\frac{8}{9} \mu_{+}
$$

Suppose in addition that

$$
\left|\left[u \leq \mu^{-}+\frac{1}{2} \omega\right] \cap Q_{\rho}(\omega)\right| \leq \nu_{-}\left|Q_{\rho}(\omega)\right|
$$

where $\nu_{-}$is defined in (4.1) with $\xi=1 / 2$ and $\theta=\omega$. Then by Lemma 4.1 with $a=\frac{1}{2}$, we have either

$$
\omega \leq 2 I_{p, \rho}
$$

or

$$
u \geq \mu^{-}+\frac{1}{4} \omega \quad \text { a.e. in } Q_{\frac{\rho}{2}}(\omega) .
$$

The latter implies

$$
\underset{Q_{\frac{\rho}{2}}(\omega)}{\operatorname{ess} \operatorname{osc}} u \leq \frac{3}{4} \omega
$$

\subsection{Case III}

As in the previous case suppose that

$$
\omega>\frac{8}{9} \mu_{+},
$$

but

$$
\left|\left[u \leq \mu^{-}+\frac{1}{2} \omega\right] \cap Q_{\rho}(\omega)\right|>\nu_{-}\left|Q_{\rho}(\omega)\right| .
$$

Then there exists some

$$
-\omega \rho^{2} \leq s \leq-\frac{1}{2} \nu_{-} \omega \rho^{2}
$$

such that

$$
\left|\left[u(\cdot, s)<\mu^{-}+\frac{1}{2} \omega\right] \cap K_{\rho}\right|>\frac{1}{2} \nu_{-}\left|K_{\rho}\right| .
$$


Indeed, if the above inequality does not hold for any $s$ in the given interval, then

$$
\begin{aligned}
\left|\left[u<\mu^{-}+\frac{1}{2} \omega\right] \cap Q_{\rho}(\omega)\right|= & \int_{-\omega \rho^{2}}^{-\frac{1}{2} \nu_{-} \omega \rho^{2}}\left|\left[u(\cdot, s)<\mu^{-}+\frac{1}{2} \omega\right] \cap Q_{\rho}(\omega)\right| d s \\
& +\int_{-\frac{1}{2} \nu_{-} \omega \rho^{2}}^{0}\left|\left[u(\cdot, s)<\mu^{-}+\frac{1}{2} \omega\right] \cap Q_{\rho}(\omega)\right| d s \\
& \leq \nu_{-}\left|Q_{\rho}(\omega)\right| .
\end{aligned}
$$

Since $\mu_{+}-\frac{1}{4} \omega>\mu^{-}+\frac{1}{2} \omega$ always holds, (5.1) implies

$$
\left|\left[u(\cdot, s)>\mu_{+}-\frac{1}{4} \omega\right] \cap K_{\rho}\right| \leq\left(1-\frac{1}{2} \nu_{-}\right)\left|K_{\rho}\right| .
$$

Based on this, we use the logarithmic estimate to show that such a measure theoretical information propagates in time.

Claim 1: There exists a positive integer $n_{*}$ depending only on $N$ such that

$$
\left|\left[u(\cdot, t)>\mu_{+}-\frac{\omega}{2^{n_{*}}}\right] \cap K_{\rho}\right| \leq\left(1-\frac{1}{4} \nu_{-}^{2}\right)\left|K_{\rho}\right| \quad \text { for all } s<t<0 .
$$

Proof. In the logarithmic estimate we take

$$
k=\mu_{+}-\frac{1}{4} \omega, \quad c=\frac{\omega}{2^{n+2}} .
$$

This gives

$$
\psi(u)=\ln ^{+}\left[\frac{H}{H-\left[u-\left(\mu_{+}-\frac{1}{4} \omega\right)_{+}\right]-\frac{\omega}{2^{n+2}}}\right]
$$

where

$$
H=\operatorname{essip}_{K_{2 \rho} \times(s, 0)}(u-k)_{+} .
$$

Choose a cutoff function $\zeta$ which satisfies $\zeta=1$ on $K_{(1-\sigma) \rho}$ and $\zeta=0$ on $\partial K_{\rho}$, such that

$$
|D \zeta| \leq \frac{1}{\sigma \rho}
$$

Hence, for all $s<t<0$,

$$
\int_{K_{(1-\sigma) \rho}(y)} \psi^{2}(u)(x, t) d x \leq \int_{K_{\rho}(y)} \psi^{2}(u)(x, s) d x+\frac{\gamma}{(\sigma \rho)^{2}} \int_{s}^{0} \int_{K_{\rho}} \frac{\psi(u)}{u} d x d t .
$$

Note that

$$
\psi \leq n \ln 2
$$

The first term on the right-hand side is estimated by

$$
\int_{K_{\rho}(y)} \psi^{2}(u)(x, s) d x \leq n^{2} \ln ^{2} 2\left(1-\frac{1}{2} \nu\right)\left|K_{\rho}\right| .
$$


The second term is estimated by

$$
\frac{\gamma}{(\sigma \rho)^{2}} \int_{s}^{0} \int_{K_{\rho}} \frac{\psi(u)}{u} d x d t \leq \frac{\gamma n}{(\sigma \rho)^{2}}\left(\omega \rho^{2}\right) \omega^{-1}\left|K_{\rho}\right| \leq \frac{\gamma n}{\sigma^{2}}\left|K_{\rho}\right| .
$$

The left-hand side is estimated below by integrating over the smaller set

$$
\left[u(\cdot, t)>\mu_{+}-\frac{\omega}{2^{n+2}}\right] \cap K_{(1-\sigma) \rho} .
$$

On such a set

$$
\psi^{2} \geq \ln ^{2}\left(\frac{\frac{\omega}{4}}{\frac{\omega}{2^{n+1}}}\right)=(n-1)^{2} \ln ^{2} 2 .
$$

Thus, combining all above estimates yields

$$
\left|\left[u(\cdot, t)>\mu_{+}-\frac{\omega}{2^{n+2}}\right] \cap K_{(1-\sigma) \rho}\right| \leq\left(\frac{n}{n-1}\right)^{2}\left(1-\frac{1}{2} \nu_{-}\right)\left|K_{\rho}\right|+\frac{\gamma}{n \sigma^{2}}\left|K_{\rho}\right|
$$

for all $s<t<0$. On the other hand,

$$
\begin{aligned}
& \left|\left[u(\cdot, t)>\mu_{+}-\frac{\omega}{2^{n+2}}\right] \cap K_{\rho}\right| \\
& \leq\left|\left[u(\cdot, t)>\mu_{+}-\frac{\omega}{2^{n+2}}\right] \cap K_{(1-\sigma) \rho}\right|+\left|K_{\rho}-K_{(1-\sigma) \rho}\right| \\
& \leq\left|\left[u(\cdot, t)>\mu_{+}-\frac{\omega}{2^{n+2}}\right] \cap K_{(1-\sigma) \rho}\right|+N \sigma\left|K_{\rho}\right| .
\end{aligned}
$$

Therefore,

$$
\left|\left[u(\cdot, t)>\mu_{+}-\frac{\omega}{2^{n+2}}\right] \cap K_{\rho}\right| \leq\left[\left(\frac{n}{n-1}\right)^{2}\left(1-\frac{1}{2} \nu_{-}\right)+\frac{\gamma}{n \sigma^{2}}+N \sigma\right]\left|K_{\rho}\right| .
$$

The claim is proved by choosing $\sigma$ so small, and then $n$ large enough.

Using the measure theoretical information obtained for every time level of the cylinder

$$
Q_{\rho}\left(\frac{1}{2} \nu_{-} \omega\right)=K_{\rho} \times\left(-\frac{1}{2} \nu_{-} \omega \rho^{2}, 0\right]
$$

in Claim 1, we are able to show

Claim 2: For any $\nu_{*} \in(0,1)$ there exists a positive integer $l$ such that

$$
\left|\left[u>\mu_{+}-\frac{\omega}{2^{n_{*}+l}}\right] \cap Q_{\rho}\left(\frac{1}{2} \nu_{-} \omega\right)\right| \leq \nu_{*}\left|Q_{\rho}\left(\frac{1}{2} \nu_{-} \omega\right)\right| .
$$

Proof. Let $Q=Q_{\rho}\left(\frac{1}{2} \nu_{-} \omega\right)$ and $Q^{\prime}=Q_{2 \rho}\left(\frac{1}{2} \nu_{-} \omega\right)$. Write down the energy estimate over $Q^{\prime}$ for

$$
\left(u-k_{j}\right)_{+} \quad \text { where } k_{j}=\mu_{+}-\frac{\omega}{2^{j}} \quad \text { for } j=n_{*}, \cdots, n_{*}+l .
$$


Choose a cutoff function $\zeta$ which satisfies $\zeta=1$ on $Q$, and vanishes on the parabolic boundary of $Q^{\prime}$, such that

$$
|D \zeta| \leq \frac{1}{\rho}, \quad\left|\zeta_{t}\right| \leq \frac{1}{\nu_{-} \omega \rho^{2}}
$$

Then keeping in mind we assumed at the beginning that $\omega>8 \mu_{+} / 9$, the energy estimate gives

$$
\omega^{-1} \iint_{Q}\left|D\left(u-k_{j}\right)_{+}\right|^{2} d x d t \leq \frac{\gamma}{\nu_{-} \omega \rho^{2}}\left(\frac{\omega}{2^{j}}\right)^{2}|Q| .
$$

Next, we apply the discrete isoperimetric inequality on page 15 of 3 to $u(\cdot, t)$ for $-\frac{1}{2} \nu_{-} \omega \rho^{2}<t<0$, over the cube $K_{\rho}$, for levels $k_{j}<k_{j+1}$. Taking into account the measure theoretical information from Claim 1, this gives

$$
\begin{aligned}
\frac{\omega}{2^{j+1}}\left|\left[u(\cdot, t)>k_{j+1}\right] \cap K_{\rho}\right| \\
\leq \frac{\gamma \rho^{N+1}}{\left|\left[u(\cdot, t)<k_{j}\right] \cap K_{\rho}\right|} \int_{\left[k_{j}<u(\cdot, t)<k_{j+1}\right] \cap K_{\rho}}|D u| d x \\
\leq \frac{\gamma}{\nu_{-}^{2}} \rho\left(\int_{\left[k_{j}<u(\cdot, t)<k_{j+1}\right] \cap K_{\rho}}|D u|^{2} d x\right)^{\frac{1}{2}} \\
\quad \times\left|\left(\left[u(\cdot, t)>k_{j}\right]-\left[u(\cdot, t)>k_{j+1}\right]\right) \cap K_{\rho}\right|^{\frac{1}{2}} .
\end{aligned}
$$

Set

$$
A_{j}=\left[u>k_{j}\right] \cap Q
$$

and integrate the above estimate in $d t$ over $-\frac{1}{2} \nu_{-} \omega \rho^{2}<t<0$; we obtain

$$
\frac{\omega}{2^{j}}\left|A_{j+1}\right| \leq \frac{\gamma}{\nu_{-}^{2}} \rho\left(\iint_{Q}\left|D\left(u-k_{j}\right)_{+}\right|^{2} d x d t\right)^{\frac{1}{2}}\left(\left|A_{j}\right|-\left|A_{j+1}\right|\right)^{\frac{1}{2}} .
$$

Now square both sides of the above inequality and use (5.2) to estimate the term containing $D\left(u-k_{j}\right)_{+}$, to obtain

$$
\left|A_{j+1}\right|^{2} \leq \frac{\gamma}{\nu_{-}^{5}}|Q|\left(\left|A_{j}\right|-\left|A_{j+1}\right|\right) .
$$

Add these inequalities from $n_{*}$ to $n_{*}+l-1$ to obtain

$$
(l-2)\left|A_{n_{*}+l}\right|^{2} \leq \sum_{j=n_{*}+1}^{n_{*}+l-1}\left|A_{j+1}\right|^{2} \leq \frac{\gamma}{\nu_{-}^{5}}|Q|^{2} .
$$

From this

$$
\left|A_{n_{*}+l}\right| \leq \frac{1}{\sqrt{l-2}} \sqrt{\frac{\gamma}{\nu_{-}^{5}}}|Q|
$$


Given a number $\nu_{*} \in(0,1)$, we can choose $l$ large enough to guarantee

$$
\frac{1}{\sqrt{l-2}} \sqrt{\frac{\gamma}{\nu_{-}^{5}}}<\nu_{*}
$$

This finishes the proof.

Now we are ready to finish Case III. Choosing $a=\frac{1}{2}, b=\frac{1}{8}$ and $\theta=\frac{1}{2} \nu_{-} \omega$, the constant $\nu_{+}$from (4.4) becomes

$$
\nu_{+}=\frac{1}{\gamma} \nu_{-}^{\frac{N}{2}}(1-\xi)^{\frac{N+2}{2}}
$$

with

$$
\xi=\frac{1}{2^{n_{*}+l}}, \quad n_{*} \text { and } l \text { to be determined. }
$$

Then after choosing $n_{*}$ from Claim 1, we can choose $l$ so large that

$$
\frac{1}{\sqrt{l-2}} \sqrt{\frac{\gamma}{\nu_{-}^{5}}}<\frac{1}{\gamma} \nu_{-}^{\frac{N}{2}}\left(1-\frac{1}{2^{n_{*}+l}}\right)^{\frac{N+2}{2}} .
$$

By Lemma 4.2, we obtain that

$$
u \leq \mu_{+}-\frac{\omega}{2^{n_{*}+l+1}} \quad \text { in } \quad Q_{\frac{\rho}{2}\left(\frac{1}{2} \nu_{-} \omega\right)} .
$$

This in turn implies

$$
\underset{Q_{\frac{\rho}{2}\left(\frac{1}{2} \nu_{-} \omega\right)}^{\operatorname{ess} \operatorname{Osc}} u \leq(1-\delta) \omega}{\operatorname{where}} \delta=\frac{1}{2^{n_{*}+l+1}} .
$$

Proof of Theorem 1.1. Combining all these cases above, we have proved that once we have

$$
\underset{Q_{\rho}(\omega)}{\operatorname{ess} \operatorname{Osc}} u \leq \omega
$$

we can find positive constants

$$
c=\min \left\{\frac{1}{2}, \sqrt{\frac{\nu_{-}}{8}}\right\} \quad \text { and } \quad \lambda=\min \left\{\frac{3}{4}, 1-\eta, 1-\delta\right\}
$$

such that

$$
\underset{Q_{c \rho}(\lambda \omega)}{\operatorname{ess} \operatorname{osc}} u \leq \max \left\{\lambda \omega, 2 I_{p, \rho}\right\}
$$

Relabel the quantities $\rho$ and $\omega$ chosen above as $\rho_{o}$ and $\omega_{o}$. Now let

$$
\omega_{1}=\max \left\{\lambda \omega_{o}, 2 I_{p, \rho_{o}}\right\} \quad \text { and } \rho_{1}=c \rho_{o}
$$

such that

$$
Q_{\rho_{1}}\left(\omega_{1}\right) \subset Q_{c \rho_{o}}\left(\lambda \omega_{o}\right) \quad \text { and } \quad \underset{Q_{\rho_{1}}\left(\omega_{1}\right)}{\operatorname{essosc}} u \leq \omega_{1}
$$


The above set inclusion is verified if

$$
\omega_{1} \rho_{1}^{2}=\max \left\{\lambda \omega_{o}, 2 I_{q, \rho_{o}}\right\}\left(c \rho_{o}\right)^{2} \leq \lambda \omega_{o}\left(c \rho_{o}\right)^{2} .
$$

This holds naturally unless

$$
\lambda \omega_{o} \leq 2 I_{p, \rho_{o}},
$$

but then there is nothing to prove since

$$
\underset{Q_{\rho_{o}}\left(\omega_{o}\right)}{\operatorname{ess} \operatorname{osc}} u \leq \frac{2}{\lambda} I_{p, \rho_{o}} .
$$

Hence, with such a choice of $c$ we now have

$$
\underset{Q_{\rho_{1}}\left(\omega_{1}\right)}{\operatorname{ess} \operatorname{osc}} u \leq \omega_{1} .
$$

According to what we have shown, one has

$$
\underset{Q_{c \rho_{1}}\left(\lambda \omega_{1}\right)}{\operatorname{ess~osc}} u \leq \max \left\{\lambda \omega_{1}, 2 I_{p, \rho_{1}}\right\} .
$$

Now define

$$
\omega_{2}=\max \left\{\lambda \omega_{1}, 2 I_{p, \rho_{1}}\right\} \quad \text { and } \quad \rho_{2}=c \rho_{1},
$$

and we want to show that

$$
Q_{\rho_{2}}\left(\omega_{2}\right) \subset Q_{c \rho_{1}}\left(\lambda \omega_{1}\right) \text { and hence } \underset{Q_{\rho_{2}}\left(\omega_{2}\right)}{\operatorname{ess} \text { osc }} u \leq \omega_{2} .
$$

The above set inclusion is verified if

$$
\omega_{2} \rho_{2}^{2}=\max \left\{\lambda \omega_{1}, 2 I_{p, \rho_{1}}\right\}\left(c \rho_{1}\right)^{2} \leq \lambda \omega_{1}\left(c \rho_{1}\right)^{2} .
$$

This holds naturally unless

$$
\lambda \omega_{1} \leq 2 I_{p, \rho_{1}},
$$

but then there is nothing to prove since

$$
\underset{Q_{\rho_{1}}\left(\omega_{1}\right)}{\operatorname{esc} \operatorname{osc}} u \leq \frac{2}{\lambda} I_{p, \rho_{1}}
$$

Iterating in this fashion, one concludes that there are positive numbers $c, \lambda \in$ $(0,1)$ such that constructing

$$
\rho_{n}=c^{n} \rho_{o} \quad \text { and } \quad \omega_{n}=\max \left\{\lambda \omega_{n-1}, 2 I_{p, \rho_{n-1}}\right\}
$$

one obtains

$$
\underset{Q_{\rho_{n}}\left(\omega_{n}\right)}{\operatorname{ess} \operatorname{osc}} u \leq \max \left\{\omega_{n}, \frac{2}{\lambda} I_{p, \rho_{n-1}}\right\}
$$

Let $0<r<\rho_{o} \leq R_{o}$ be fixed. Since the sequence $\left\{\omega_{n} \rho_{n}^{2}\right\}$ is strictly decreasing and gives a partition of the interval $\left(0, \omega_{o} \rho_{o}^{2}\right)$, there must be some positive integer $n$ such that

$$
\rho_{n+1}^{2} \omega_{n+1} \leq r^{2} \omega_{o}<\rho_{n}^{2} \omega_{n}
$$


Noting that

$$
\omega_{n} \leq \omega_{o} \text { and } \omega_{n+1} \geq \lambda^{n+1} \omega_{o},
$$

this implies that $r<\rho_{n}$ and

$$
n+1 \geq \frac{\ln \left(\frac{r}{\rho_{o}}\right)}{\ln (\sqrt{\lambda} c)} .
$$

Then it is not hard to see that

$$
Q_{r}\left(\omega_{o}\right) \subset Q_{\rho_{n}}\left(\omega_{n}\right)
$$

and either

$$
\underset{Q_{r}\left(\omega_{o}\right)}{\operatorname{ess} \operatorname{osc}} u \leq \omega_{n} \quad \text { or } \quad \omega_{n-1} \leq \frac{2}{\lambda} I_{p, \rho_{n-1}} .
$$

Note that

$$
\begin{aligned}
\omega_{n} & =\max \left\{\lambda^{n} \omega_{o}, 2 \lambda^{n-1} I_{p, \rho_{o}}, \cdots, 2 \lambda I_{p, \rho_{n-2}}, 2 I_{p, \rho_{n-1}}\right\} \\
& \leq \lambda^{n} \omega_{o}+\frac{2}{1-\lambda} I_{p, \rho_{o}} .
\end{aligned}
$$

Here we made the convention that the function $\rho \rightarrow I_{p, \rho}$ is non-decreasing. Otherwise, one could use

$$
\tilde{I}_{p, \rho}=\sup _{0<\tau<\rho} I_{p, \tau}
$$

Thus, there is some $\alpha \in(0,1)$ depending only on the data such that

$$
\underset{Q_{r}\left(\omega_{o}\right)}{\operatorname{ess} \operatorname{Osc}} u \leq \lambda^{n} \omega_{o}+C I_{p, \rho_{o}} \leq \bar{C}\left[\omega_{o}\left(\frac{r}{\rho_{o}}\right)^{\alpha}+I_{p, \rho_{o}}\right]
$$

where

$$
\alpha=\frac{\ln \lambda}{\ln (\sqrt{\lambda} c)} \quad \text { and } \quad C=\max \left\{\frac{2}{1-\lambda}, \frac{2}{\lambda}\right\} .
$$

Now choose $\rho_{o}=R_{o}^{1-\mu} r^{\mu}$, and conclude we have

$$
\underset{Q_{r}\left(\omega_{o}\right)}{\operatorname{ess} \operatorname{Osc}} u \leq \bar{C}\left[\omega_{o}\left(\frac{r}{R_{o}}\right)^{(1-\mu) \alpha}+I_{p, R_{o}^{1-\mu}} r^{\mu}\right] .
$$

\section{References}

[1] P. Daskalopoulos and M. del Pino, On a singular diffusion equation, Comm. Anal. Geom., 3(3-4), (1995), 523-542.

[2] P. Daskalopoulos and M. del Pino, On the Cauchy problem for $u_{t}=\Delta \log u$ in higher dimensions, Math. Ann., 313, (1999), 189-206.

[3] E. DiBenedetto, "Degenerate Parabolic Equations", Universitext, SpringerVerlag, New York, 1993. 
[4] E. DiBenedetto and D. J. Diller, About a singular parabolic equation arising in thin film dynamics and in the Ricci flow for complete $\mathbb{R}^{2}$, in "Partial Differential Equations and Applications", Lecture Notes in Pure and Appl. Math., 177, Dekker, New York, (1996), 103-119.

[5] E. DiBenedetto and D. J. Diller, Singular semilinear elliptic equations in $L^{1}\left(\mathbb{R}^{N}\right)$, in "Topics in nonlinear analysis", Progr. Nonlinear Differential Equations Appl., 35, Birkhäuser, Basel, (1999), 143-181.

[6] E. DiBenedetto, U. Gianazza and N. Liao, On the local behavior of nonnegative solutions to a logarithmically singular equation, Discrete Contin. Dyn. Syst. Ser. B , 17(6), 2012, 1841-1858.

[7] E. DiBenedetto, U. Gianazza and N. Liao, Logarithmically singular parabolic equations as limits of the porous medium equation, Nonlinear Anal., 75(12), (2012), 4513-4533.

[8] E. DiBenedetto, U. Gianazza and N. Liao, Two remarks on the local behavior of logarithmically singular diffusion equations, Riv. Math. Univ. Parma (N.S.), 5(1), (2014), 139-182.

[9] E. DiBenedetto, U. Gianazza and V. Vespri, "Harnack's Inequality for Degenerate and Singular Parabolic Equations", Springer Monographs in Mathematics, Springer-Verlag, New York, 2012.

[10] L. C. Evans and R. Gariepy, "Measure Theory and Fine Properties of Functions", Studies in Advanced Mathematics, CRC Press, 1992.

[11] K. M. Hui, On some Dirichlet and Cauchy problems for a singular diffusion equation, Differential Integral Equations, 15(7), (2002), 769-804.

[12] S. Y. Hsu, Global existence and uniqueness of solutions of the Ricci flow equation, Differential Integral Equations, 14(3), (2001), 305-320.

[13] O. A. Ladyzhenskaya, V. A. Solonnikov, and N. N. Ural'tseva, "Linear and Quasilinear Equations of Parabolic Type", Translations in Mathematical Monographs, Vol. 23, American Mathematical Society, Providence, R.I., 1968.

[14] N. Liao, Existence and non-existence of solutions to a logarithmic diffusion equation in bounded domains, Manuscripta Math., 147(1), (2015), 101-138.

[15] J. L. Vázquez, Failure of the strong maximum principle in nonlinear diffusion. existence of needles, Comm. Partial Differential Equations, 30(7-9), (2005), 1263-1303.

[16] J. L. Vázquez, Nonexistence of solutions for nonlinear heat equations of fast-diffusion type, J. Math. Pures Appl., 71(9), (1992), 503-526.

[17] M. B. Williams and S. H. Davis, Nonlinear theory of film rupture, Jour. of Colloid and Interface Sc., 90(1), (1982), 220-228. 
[18] L. Wu, The Ricci flow on complete $\mathbb{R}^{2}$, Comm. Anal. Geom. 1, (1993), 439-472.

Naian Liao

College of Mathematics and Statistics

Chongqing University

Chongqing, China, 401331

email: 1iaon@cqu.edu.cn 